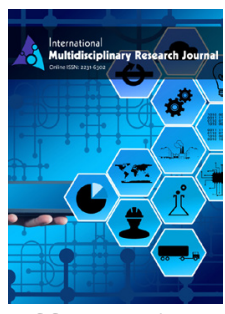

ISSN: 2231-6302

Received: April 22, 2020 Accepted: June 08, 2020 Published: June 19, 2020

*Corresponding Author: Viral M. Vaghela Email: viraldncs@gmail.com

\section{Glimpse of avian diversity near Mithiviradi Village, Bhavnagar District, Gujarat, India}

\author{
Viral M. Vaghela*, Pravinsang P. Dodia \\ 'Zoology Departement, Sir P. P. Institute of Science, MK Bhavnagar University, Bhavnagar - 364001, Gujarat, India,
}

\begin{abstract}
Birds are very good biological indicators of environment health. Changes in various activity of bird can be used to check the long term effects of changes in habitat. The study was conducted to find out the diversity of birds in and around Mithiviradi village (21030'06” N; 72¹3'49”E) located in Talaja tehsil, Bhavnagar, Gujarat. The avian diversity was surveyed during Jan. 2016 to Jan. 2018. During the survey total 104 bird species were recorded out of this 78 species were residential and breeding, 15 species were migratory, and 09 were resident, breeding and migratory. This avian diversity represents 16 orders and 50 families.
\end{abstract}

KEYWORDS: Mithiviradi, Avian diversity, Bird species, Residential, Breeding, Migratory, Common

\section{INTRODUCTION}

Bhavnagar District is situated at coastal region of Gulf of Khambhat. Mithiviradi village is $40 \mathrm{~km}$ away from Bhavnagar city. Mithiviradi village and their surrounding area were selected for the proposed Nuclear power station but at present it was postponed. The area is having various kind of habitat like agricultural, gauchar, forest patch, coastal area and temporary water body (seasonal river and check dam) all these provide suitable residence to diversity of birds and make the area rich in avian diversity. Birds play key role in pollination and seed dispersal [1]. India is having a total of 1263 species of birds which constituting about $12 \%$ of the world avifauna [2], whereas Gujarat has 574 bird species [3]. Rich avifaunal diversity of Gujarat is due to various types of habitats like, deciduous and thorn forests, grasslands, wetlands, marine intertidal areas, scrublands and saline deserts [4]. Bird diversity of Gujarat state and particularly of coastal region of Saurashtra has low threats. But some remote areas are still not surveyed with reference to status of their bird diversity. Mithiviradi is situated in Talaja tehsil $40 \mathrm{~km}$ away from Bhavnagar District headquarter and $15 \mathrm{~km}$ from Alang ship breaking yard near the coast of Gulf of Khambhat.

\section{Study Area}

The study was conducted to find out the diversity of birds in and around Mithiviradi village (21030'06" N; 72'13'49”E) located in Talaja tehsil, Bhavnagar, Gujarat, India. Area covers with the gauchar land, agriculture farm, wet lands and coastal part of gulf of Khambhat.

\section{MATERIALS AND METHODS}

The study was carried out during January 2016 to January 2018. The area was visited once in a month, hence, 4 times in one season. Data was collected by point count method for that some points are selected in all habitat. All observations were taken during morning (06:30hrs. to 09:30 hrs.) as well as evening (15:30 hrs. to 18:30 hrs.) when birds are found most active. 8X40 Nikon binocular was used for observation and Sony HX 400 camera was used for documentation. Identification of the bird species was confirmed by the book of Indian Birds [5], Birds of the Indian Subcontinent [6] and different volume of Raol [7-9]. For the status of bird we follow A Checklist of the Birds of Gujarat [2], [10]. Annual temperature of the study area ranges between $27^{\circ} \mathrm{C}$ and $42^{\circ} \mathrm{C}$ and average annual rainfall is $580 \mathrm{~mm}$ (Metrology station Bhavnagar).

\section{RESULT AND DISCUSSION}

During the study total 104 bird species were recorded out of this 78 species are residential and breeding, 15 species are migratory, 09 species are residential breeding and migratory, 01 species is resident and 01 species is passage migrant (Table 1 ). These bird species represents 16 orders and 50 families (Fig. 1). Result also 
Table 1: List of birds found near Mithiviradi village Gujarat, India.

\begin{tabular}{|c|c|c|c|c|c|c|}
\hline Sr. no. & Order & Family & Common Name & Scientific Name & Res.-Status & Cons.Status \\
\hline 1 & Podicipedi-formes & Podicipididae & Little Grebe & Tachybaptus ruficollis & $\mathrm{RB}$, & Common \\
\hline 2 & Pelicani-formes & Anhinginae & Darter & Anhinga melanogaster & $\mathrm{RB}$, & Uncommon \\
\hline 3 & & Phalacrocoracidae & Great Cormorant & Phalacrocorax carbo & $\mathrm{RB}$ & Common \\
\hline 4 & & & Little Cormorant & Phalacrocorax niger & RB & Common \\
\hline 5 & Ciconii-formes & Ardeidae & Black-crowned Night Heron & Nycticorax nycticorax & RB & Common \\
\hline 6 & & & Cattle Egret & Bulbulcus ibis & RB & Common \\
\hline 7 & & & Great Egret & Casmerodius albus & $\mathrm{RB}$ & Common \\
\hline 8 & & & Grey Heron & Ardea cinerea & $\mathrm{RB}$ and $\mathrm{M}$ & Common \\
\hline 9 & & & Indian Pond Heron & Ardeola grayii & RB & Common \\
\hline 10 & & & Intermediate Egret & Mesophoyx intermedia & RB & Common \\
\hline 11 & & & Little Egret & Egretta garzetta & RB & Common \\
\hline 12 & & & Purple Heron & Ardea purpurea & RB & Common \\
\hline 13 & & & Western Reef Egret & Egretta gularis & $\mathrm{RB}$ and $\mathrm{M}$ & Common \\
\hline 14 & & Ciconidae & Painted Stork & Mycteria leucocephala & RB & Common \\
\hline 15 & & Threskiornithidae & Red-naped Ibis & Pseudibis papillosa & RB & Common \\
\hline 16 & & & Black-headed Ibis & Threskiornis melanocephalus & $\mathrm{RB}$ & Common \\
\hline 17 & & Phoenicopteridae & Greater Flamingo & Phoenicopterus roseus & $\mathrm{RB}$ and $\mathrm{M}$ & Common \\
\hline 18 & & Anatidae & Knob-billed Duck & Sarkidiornis melanotos & $\mathrm{RB}$ & Common \\
\hline 19 & & & Indian Spot-Billed Duck & Anas poecilorhyncha & RB & Common \\
\hline 20 & Falconiformes & Accipitridae & Black Kite & Milvus migrans & RB & Common \\
\hline 21 & & & Black-winged Kite & Elanus caeruleus & RB & Common \\
\hline 22 & & & Brahminy Kite & Haliastur indus & RB & Common \\
\hline 23 & & & Shikra & Accipiter badius & RB & Common \\
\hline 24 & & & Oriental Honey Buzzard & Pernis ptilorhyncus & $\mathrm{RB}$ and $\mathrm{M}$ & Common \\
\hline 25 & & & Eurasian Marsh Harrier & Circus aeruginosus & $\mathrm{M}$ & Common \\
\hline 26 & & Falconidae & Common Kestrel & Falco tinnunculus & $\mathrm{M}$ & Common \\
\hline 27 & Galliformes & Phasianidae & Grey Francolin & Francolinus pondicerianus & RB & Common \\
\hline 28 & & & Painted Francolin & Francolinus pictus & RB & Common \\
\hline 29 & & & Indian Peafowl & Pavo cristatus & $\mathrm{RB}$ & Common \\
\hline 30 & & & Rain Quail & Coturnix coromandelica & $\mathrm{RB}$ and $\mathrm{M}$ & Common \\
\hline 31 & Gruiformes & Gruidae & Demoiselle Crane & Grus virgo & $\mathrm{M}$ & Common \\
\hline 32 & & Rallidae & Eurasian Coot & Fulica atra & RB & Common \\
\hline 33 & & & Purple Swamphen & Porphyrio porphyrio & RB & Common \\
\hline 34 & & & White-Breasted Waterhen & Amaurornis phoenicurus & RB & Common \\
\hline 35 & Charadrii-formes & CharadriIdae & Red-Wattled Lapwing & vanellus indicus & RB & Common \\
\hline 36 & & & Little Ringed Plover & Charadrius dubius & RB & Common \\
\hline 37 & & Turnicidae & Barred Buttonquail & Turnix suscitator & $\mathrm{R}$ & Common \\
\hline 38 & & Scolopacidae & Black-Tailed Godwit & Limosa limosa & M & Common \\
\hline 39 & & & Common Sandpiper & Actitis hypoleucos & M & Common \\
\hline 40 & & & Eurasian Curlew & Numenius arquata & M & Common \\
\hline 41 & & & Ruff & Philomachus pugnax & M & Common \\
\hline 42 & & Burhinidae & Indian Thick-Knee & Burhinus (oedicnemus) indicus & RB & Common \\
\hline 43 & & Recurvirostridae & Black-Winged Stilt & Himantopus himantopus & RB & Common \\
\hline 44 & & Laridae & Caspian Gull & Larus cachinnans & M & Rare \\
\hline 45 & & & River Tern & Sterna aurantia & RB & Common \\
\hline 46 & Columbi-formes & Columbidae & Eurasian Collared Dove & Streptopelia decaocto & RB & Common \\
\hline 47 & & & Laughing Dove & Streptopelia senegalensis & RB & Common \\
\hline 48 & & & Red-collared Dove & Streptopelia tranquebarica & RB & Common \\
\hline 49 & & & Common Pigeon & Columba livia & RB & Common \\
\hline 50 & & & Yellow-Footed Green Pigeon & Treron phoenicopterus & RB & Common to Uncommon \\
\hline 51 & Psittaci-formes & Psittacidae & Rose-Ringed Parakeet & Psittacula krameri & RB & Common \\
\hline 52 & & & Plum headed Parakeet & Psittacula cyanocephala & RB & Common \\
\hline 53 & Cuculiformes & Cuculidae & Common Hawk Cuckoo & Hierococcyx varius & $\mathrm{RB}$ and $\mathrm{M}$ & Common \\
\hline 54 & & & Greater Coucal & Centropus (sinensis) parroti & $\mathrm{RB}$ & Common \\
\hline 55 & & & Jacobin Cuckoo & Clamator jacobinus & $\mathrm{RB}$ and $\mathrm{M}$ & Common \\
\hline 56 & & & Sirkeer Malkoha & Taccocua leschenaultia & RB & Uncommonto rare \\
\hline 57 & & & Asian -Koel & Eudynamys scolopaceus & RB & Common \\
\hline 58 & Strigiformes & Tytonidae & Barn Owl & Tyto alba & RB & Common to uncommon \\
\hline 59 & & Strigidae & Spotted Owlet & Athene brama & RB & Common \\
\hline 60 & Caprimulgi-formes & Caprimulgidae & Indian Nightjar & Caprimulgus asiaticus & RB & Common \\
\hline 61 & Apodiformes & Apodidae & Little Swift & Apus affinis & RB & Common \\
\hline 62 & Coraciiformes & Alcedinidae & Common Kingfisher & Alcedo atthis & RB & Common \\
\hline 63 & & & Pied Kingfisher & Ceryle rudis & RB & Common \\
\hline 64 & & & White-Throated Kingfisher & Halcyon smyrnensis & RB & Common \\
\hline 65 & & Meropidae & Green Bee-Eater & Merops orientalis & $\mathrm{RB}$ & Common \\
\hline 66 & & Coraciidae & European Roller & Coracias garrulus & PM & Common to uncommon \\
\hline
\end{tabular}


Table 1: (Continued)

\begin{tabular}{|c|c|c|c|c|c|c|}
\hline Sr. no. & Order & Family & Common Name & Scientific Name & Res.-Status & Cons.Status \\
\hline 67 & & & Indian Roller & Coracias benghalensis & RB & Common \\
\hline 68 & & Upupidae & Common Hoopoe & Upupa epops & M & Common \\
\hline 69 & Piciformes & Capitonidae & Coppersmith Barbet & Megalaima haemacephala & RB & Common \\
\hline 70 & & Picidae & $\begin{array}{l}\text { Lesser Golden back } \\
\text { Woodpecker }\end{array}$ & Dinopium benghalense & RB & Common \\
\hline 71 & Passeri-formes & Alaudidae & Ashy-Crowned Sparrow Lark & Eremopterix griseus & RB & Common \\
\hline 72 & & & Crested Lark & Galerida cristata & RB & Common \\
\hline 73 & & Hirundinidae & Red-Rumped Swallow & Hirundo daurica & M & Common \\
\hline 74 & & & Wire-Tailed Swallow & Hirundo smithii & $\mathrm{RB}$ & Common \\
\hline 75 & & Laniidae & Bay-backed Shrike & Lanius vittatus & RB & Common \\
\hline 76 & & & Long-Tailed Shrike & Lanius schach & $\mathrm{RB}$ & Common \\
\hline 77 & & Oriolidae & Eurasian Golden Oriole & Oriolus (oriolus) kundoo & $\mathrm{RB}$ and $\mathrm{M}$ & Common \\
\hline 78 & & Dicruridae & Black Drongo & Dicrurus macrocercus & RB & Common \\
\hline 79 & & Sturnidae & Bank Myna & Acridotheres ginginianus & RB & Common \\
\hline 80 & & & Common Myna & Acridotheres tristis & RB & Common \\
\hline 81 & & & Rosy Starling & Pastor roseus & M & Common \\
\hline 82 & & & Brahminy Starling & Sturnia pagodarum & RB & Common \\
\hline 83 & & Corvidae & House Crow & Corvus splendens & RB & Common \\
\hline 84 & & & Indian Jungle Crow & $\begin{array}{l}\text { Corvus (macrorhynchos) } \\
\text { culminatus }\end{array}$ & RB & Common \\
\hline 85 & & & Rufous Treepie & Dendrocitta vagabunda & RB & Common \\
\hline 86 & & Campephagidae & Small Minivet & Pericrocotus cinnamomeus & RB & Common \\
\hline 87 & & Irenidae & Common Iora & Aegithina tiphia & RB & Common \\
\hline 88 & & Pycnonotidae & Red-Vented Bulbul & Pycnonotus cafer & RB & Common \\
\hline 89 & & Timaliinae & Common Babbler & Turdoides caudata & RB & Common \\
\hline 90 & & & Large Grey Babbler & Turdoides malcolmi & RB & Common \\
\hline 91 & & Monarchidae & Asian-Paradise Flycatcher & Terpsiphone paradisi & $\mathrm{RB}$ and $\mathrm{M}$, & Common \\
\hline 92 & & & Plain Priniya & Prinia inornata & RB & Common \\
\hline 93 & & & Ashy Prinia & Prinia socialis & RB & Common \\
\hline 94 & & & Common Tailor Bird & Orthotomus sutorius & $\mathrm{RB}$ & Common \\
\hline 95 & & Turdinae & Common Stonechat & Saxicola torquatus & M & Common \\
\hline 96 & & & Indian Robin & Saxicoloides fulicatus & RB & Common \\
\hline 97 & & & Oriental Magpie Robin & Copsychus saularis & $\mathrm{RB}$ & Common \\
\hline 98 & & Motacillidae & Citrine Wagtail & Motacilla citreola & $\mathrm{M}$ & Common \\
\hline 99 & & & White Wagtail & Motacilla alba & M & Common \\
\hline 100 & & & Yellow Wagtail & Motacilla flava & M & Common \\
\hline 101 & & Nectariniidae & Purple Sunbird & Cinnyris asiaticus & RB & Common \\
\hline 102 & & Passerinae & House Sparrow & Passer domesticus & RB & Common \\
\hline 103 & & Ploceinae & Baya Weaver & Ploceus philippinus & RB & Common \\
\hline 104 & & Estrildidae & Indian Silver bill & Euodice malabarica & $\mathrm{RB}$ & Common \\
\hline
\end{tabular}

$\mathrm{RB}=$ Resident Breeder, $\mathrm{R}=$ Resident, $\mathrm{W}=$ Winter Visitor, $\mathrm{RB}$ and $\mathrm{W}=$ Resident Breeder and winter visitor and PM $=$ Passage Migrant

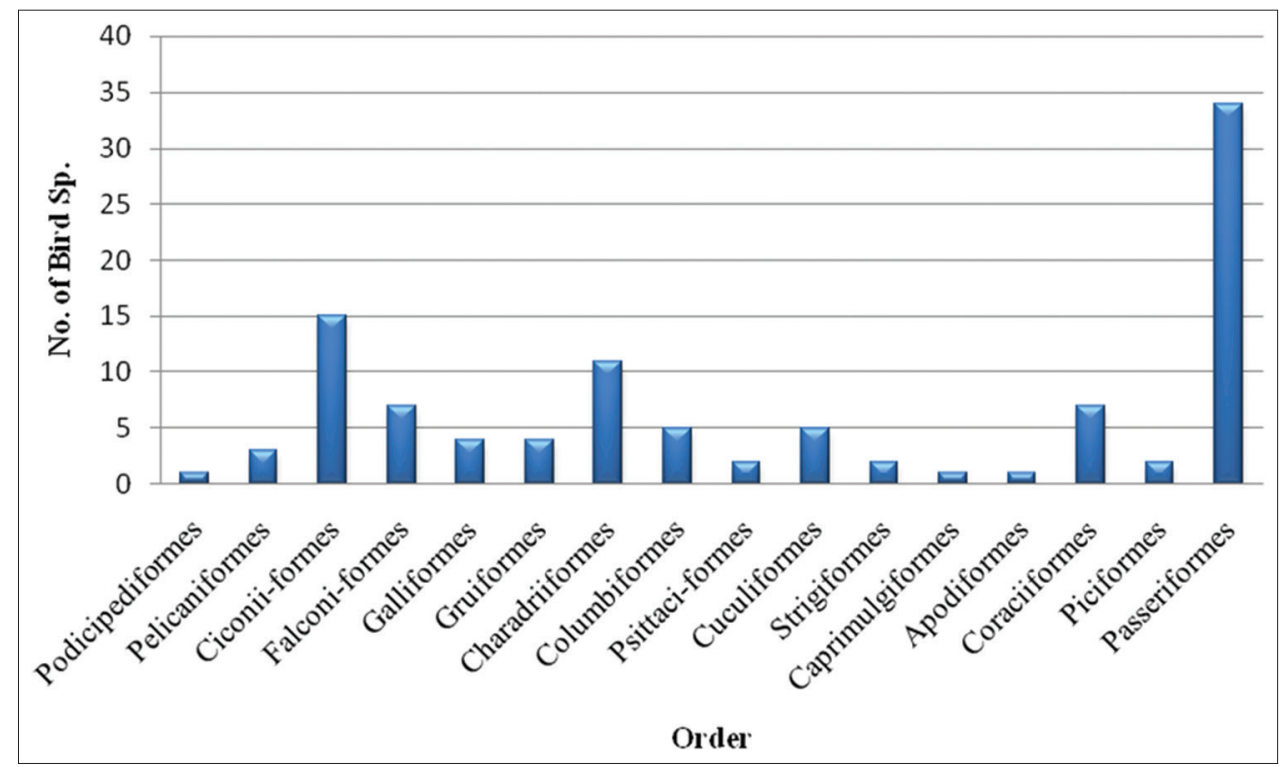

Figure 1: Graph representing relative diversity of observed bird species near Mithiviradi village 


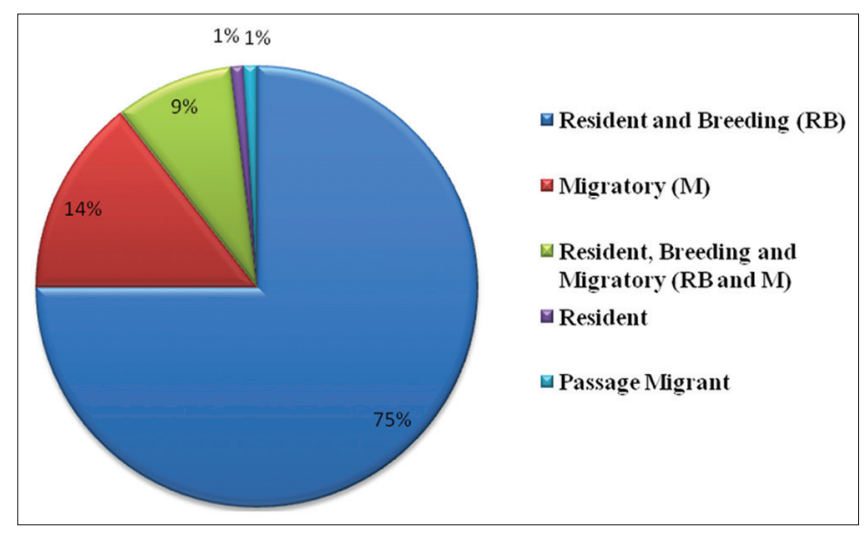

Figure 2: Chart shows the residential status of bird species found near Mithiviradi village

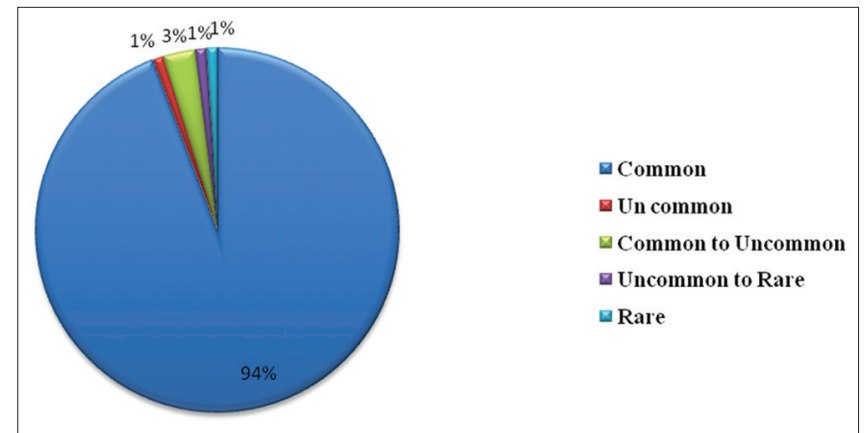

Figure 3: Chart shows the conservation status of bird species found near Mithiviradi village

indicates that 71 bird species was terrestrial and 33 bird species was observed aquatic. Maximum 34 bird species are belongs to order Passeriformes after that Ciconiformes order having 15 bird species and three orders having only 01 bird species each. It was observed that Passeriformes order also having maximum 18 families, where as Charadriiformes order having 06 families, 07 bird order having only 01 family in the study area. Ardeidae family has maximum 09 species. Result shows that the study area harbours good avian diversity and the habitat is suitable for resident and breeding birds, birds observed in the area are common (98) in their conservational status. Out of 71 terrestrial bird species found in the study area 60 species are resident and breeding, so protection of the area is helpful for breeding and foraging of these resident birds (Fig. 2). The 104 bird species observed during the study belonging to 16 orders and 50 families constitute $8.12 \%$ species, $46.72 \%$ families and $69.56 \%$ orders of Indian avifaunal diversity (Fig. 3). When compared with bird diversity of Gujarat state that is $18.12 \%$ of species. Habitat of the area mainly supports Resident and breeding species of birds but some migratory species also enjoy their visit. Baraiya and Dodia [11] recorded 100 bird species from 16 orders and 47 families near Devgana and their surrounding area in Bhavnagar district. 223 species from 61 deferent families of birds were recorded from Victoria park reserve forest area and Bortalav wetland of Bhavnagar city [12].

\section{CONCLUSION}

Present study indicates that the Mithiviradi and their surrounding area have the rich avifaunal diversity. Area also having the good vegetation covers which provided the food and shelter for the birds and other animal. Here we recorded total 104 bird species from different 50 families and 16 orders out of this $75 \%$ species were resident and breeding. Data represent the biological richness of the study area. Some migratory bird species are also enjoyed their visit during winter.

\section{ACKNOWLEDGEMENT}

We are thanks full to Dr. A.H. Shukla (HoD) Zoology department, Sir P.P. Institute of Science, MK Bhavnagar University, Mr. V. Z. Bhatti Principal, Shri Mithiviradi primary school and all the persons those who are helping us for this research.

\section{REFERENCES}

1. Bibi, F. \& Ali, Z. 2013. Measurement of diversity indices of avian communities at Taunsa Barrage Wildlife Sanctuary, Pakistan. Journal of Animal and Plant Science 23:469-474.

2. Praveen, J., Jaypal, R. \& Pittie, A. 2016. A checklist of the birds of India. Indian birds 11 (5\&6): 113-172.

3. Ganpule P. 2016. The Birds of Gujarat: Status and Distribution. Flamingo 8(3) - 12(4): 2-40.

4. Singh, H. S. 2001. Natural heritage of Gujarat (forests and wildlife). Gandhinagar, India: GEER Foundation.

5. Ali, Salim 1996. The book of Indian Birds (12 $2^{\text {th }}$ revised edition), Bombay Natural History Society, Bombay, and pp: 354.

6. Grimmett R., Inskipp C., Inskipp T. 1998. Birds of the Indian Subcontinent, Oxford University Press, Delhi, p: 384.

7. Raol, L 1991. Jal Ane Jalashayna Pankhio Panina Sangathi. Paryavaran Shikshan Kendra, Amdavad. Pp. 267.

8. Raol, L 1998. Van Upvanna Pankhi. Paryavaran Shikshan Kendra, Amdavad. Pp. 146.

9. Raol, L 2000. Vid Vagadana Pankhi. Paryavaran Shikshan Kendra, Amdavad. Pp. 224

10. B. M. Parasharya, C.K. Borad and D.N. Rank 2004. A Checklist of the Birds of Gujarat. Bird Conservation Society, Gujarat, Pp.26.

11. Harindra Baraiya and P. P. Dodia. 2016. Avian Diversity of Devgana and Surrounding Areas, Dist. Bhavnagar, Gujarat. Recent Trends in Science and Technology: vol. 1, 227-236

12. P.P. Dodia and N. H. Chavda 2012. Birds of Victoria Park reserve forest and Bortalav water reservoir in Bhavnagar, Gujarat. India. Life science leaflets 2:1-11, 2012 